\title{
Robustness of Several Estimators of the ACF of AR(1) Process With Non-Gaussian Errors
}

\author{
AA. Smadi \\ Yarmouk University, Irbid, Jordan, asmadi@yu.edu.jo \\ JJ. Jaber \\ University of Jordan, Aqaba \\ A G. Al-Zu'bi \\ Yarmouk University, Irbid, Jordan
}

Follow this and additional works at: http://digitalcommons.wayne.edu/jmasm

Part of the Applied Statistics Commons, Social and Behavioral Sciences Commons, and the Statistical Theory Commons

\section{Recommended Citation}

Smadi, A A.; Jaber, J J.; and Al-Zu'bi, A G. (2014) "Robustness of Several Estimators of the ACF of AR(1) Process With Non-Gaussian Errors," Journal of Modern Applied Statistical Methods: Vol. 13 : Iss. 1 , Article 10.

DOI: $10.22237 /$ jmasm/1398917340

Available at: http://digitalcommons.wayne.edu/jmasm/vol13/iss1/10

This Regular Article is brought to you for free and open access by the Open Access Journals at DigitalCommons@WayneState. It has been accepted for inclusion in Journal of Modern Applied Statistical Methods by an authorized editor of DigitalCommons@WayneState. 


\section{Robustness of Several Estimators of the ACF of AR(1) Process With Non-Gaussian Errors}

\author{
A. A. Smadi \\ Yarmouk University \\ Irbid, Jordan
}

\author{
J. J. Jaber \\ University of Jordan \\ Aqaba, Jordan
}

\author{
A. G. Al-Zu'bi \\ Yarmouk University \\ Irbid, Jordan
}

The autocorrelation function (ACF) plays an important role in the context of ARMA modeling, especially for their identification and estimation. This study considers the robust estimation of the ACF of the AR(1) model if the white noise (WN) process is nonGaussian. Three estimators including the ordinary moment estimator and two other (robust) estimators are considered. The impacts of the deviation from normality of the WN process on those estimators in terms of bias, MSE and distribution via Monte-Carlo simulation are examined. The empirical distribution of those estimators when the errors are normal, $t$, Cauchy and exponential are studied. Results show that the moment estimator is more affected by the change of the white noise distribution than other considered estimators.

Keywords: autocorrelation function, robust estimation, Monte-Carlo simulation, kernel density estimation

\section{Introduction}

A time series (TS) can be defined as a sequence of observations taken sequentially in time. Time series can be observed in different fields; for example, in agriculture, business, engineering and medical studies. The list of areas in which time series is observed, studied and analyzed is endless. A major feature in the development of time series is an assumption of some form of statistical equilibrium, or known as stationarity. There are two types of stationarity; the first is called strict stationarity, and the other type is called weak stationarity. In practice, it is very difficult to examine time series being strictly stationary. Further, a stochastic process $\left\{X_{t}\right\}$ is weak stationary if its mean is constant and the auto-

Dr. Smadi is in the Department of Statistics.Email him at: asmadi@yu.edu.jo.Dr.Jaber is in the Department of Risk Management \& Insurance. Dr. Al-Zu'bi is in the Department of Statistics. 


\section{ROBUSTNESS OF ESTIMATORS OF THE ACF OF AR(1) PROCESS}

covariance function (ACVF) depends on the time lag only, i.e., $\operatorname{Cov}\left(X_{t}, X_{t+k}\right)=\gamma_{k}$ as well as its $\operatorname{ACF} \rho_{k}=\gamma_{k} / \gamma_{0}$. For more details on the ACVF and ACF of stationary time series and their properties, see Wei (2006, p. 12).

The class of autoregressive moving average (ARMA) models is widely known for modeling stationary time series (Wei, 2006, p. 56-64). The stochastic process $\left\{X_{t}\right\}$ is said to follow the $\operatorname{ARMA}(p, q)$ model if:

$$
X_{t}=\phi_{1} X_{t-1}+\cdots+\phi_{p} X_{t-p}+u_{t}-\theta_{1} u_{t-1}-\cdots-\theta_{q} u_{t-q}
$$

where $\phi_{1}, \ldots, \phi_{p}$ and $\theta_{1}, \ldots, \theta_{q}$ are the AR and MA parameters, respectively, and $u_{t}$ is the white noise ( $\left.\mathrm{WN}\right)$ process, assumed iid $\left(0, \sigma_{u}^{2}\right)$ and usually assumed normal. A detailed account on ARMA models, their autocorrelation functions and building methodology is found in Box, et al. (1994).

Beside the mixed ARMA model, the $\operatorname{ARMA}(p, q)$ models also include as special cases the pure AR and pure MA models when $q=0$ and $p=0$, respectively. In particular, the pure AR(1) model is given by:

$$
X_{t}=c+\phi X_{t-1}+u_{t}
$$

which is stationary if $|\phi|<1$ (Wei, 2006).

The ACF plays an important role in the Box and Jenkins methodology for building ARMA models, especially for the identification and estimation of those models (Wei, 2006). In fact, there are other identification tools for the ARMA models, including the inverse ACF method (Cleveland, 1972); Akaike information criterion (AIC) (Akaike, 1974); the R and S array method (Gray, et al., 1978) and the corner method (Beguin, et al., 1980).

Consider the robust estimation of the ACF of AR(1) model if the WN process $\left\{u_{t}\right\}$ is non-Gaussian. Berkoun, et al. (2003) investigated robust inference for serial correlation in $\mathrm{AR}(1)$ process in the presence of a single additive outlier. Assuming that $\left\{X_{1}, \ldots, X_{n}\right\}$ is a time series following the zero-mean $\mathrm{AR}(1)$ model contaminated with a single additive outlier, they investigated three estimators of $\rho_{1}$, namely: 


\section{SMADI ET AL}

$$
\begin{gathered}
r_{1}=\frac{\sum_{t=2}^{n} Z_{t} Z_{t-1}}{\sum_{t=2}^{n} Z_{t-1}^{2}} \\
\rho_{1}^{*}=\operatorname{Med}\left\{\frac{Z_{2}}{Z_{1}}, \frac{Z_{3}}{Z_{2}}, \ldots, \frac{Z_{n}}{Z_{n-1}}\right\}
\end{gathered}
$$

and

$$
\tilde{\rho}_{1}=\frac{\operatorname{Med}\left\{Z_{1} Z_{2}, Z_{2} Z_{3}, \ldots, Z_{n-1} Z_{n}\right\}}{\operatorname{Med}\left\{Z_{1}^{2}, Z_{2}^{2}, \ldots, Z_{n-1}^{2}\right\}}
$$

where $Z_{t}=X_{t}-\bar{X}$ are the mean-subtracted data, Med(.) stands for the median, $r_{1}$ is the ordinary moment estimator of $\rho_{1}$ whereas $\rho_{1}^{*}$ and $\tilde{\rho}_{1}$ are two robust estimators of $\rho_{1}$ originally proposed by Hurwicz (1950) and Haddad (2000), respectively. Berkoun, et al. (2003) showed that the inference of $\rho_{1}$ based on $r_{1}$ is highly sensitive to a single additive outlier.

Smadi, et al. (2009) generalized these estimators for the periodic AR(1) model. They again observed that the counterpart of $r_{1}$ is more sensitive to additive outliers than other estimators.

For higher time lags $k=1,2, \ldots$ the estimators in (2) - (4) generalize to estimate $\rho_{k}$ as follows:

$$
\begin{gathered}
r_{k}=\frac{\sum_{t=k+1}^{n} Z_{t} Z_{t-k}}{\sum_{t=2}^{n} Z_{t-1}^{2}} \\
\rho_{k}^{*}=\operatorname{Med}\left\{\frac{Z_{k+1}}{Z_{1}}, \frac{Z_{k+2}}{Z_{2}}, \ldots, \frac{Z_{n}}{Z_{n-k}}\right\}
\end{gathered}
$$

and 


$$
\tilde{\rho}_{k}=\frac{\operatorname{Med}\left\{Z_{1} Z_{k+1}, Z_{2} Z_{k+2}, \ldots, Z_{n-k} Z_{n}\right\}}{\operatorname{Med}\left\{Z_{1}^{2}, Z_{2}^{2}, \ldots, Z_{n-k}^{2}\right\}}
$$

In this research, the main objective is to study the statistical properties; namely the mean, variance as well as the distribution of various estimators of $\rho_{k}$. This study is restricted to the AR(1) model as (1) along various distributions for the $\mathrm{WN}$ process. Therefore, it focuses on the robustness of estimators above subject to the distribution of the $\mathrm{WN}$ process.

In the literature of time series analysis, the area of robust inference has found considerable attention. Denby and Martin (1979) proposed the generalized M-estimates for autoregressive processes and Bustos and Yohai (1986) took the auto-covariance structure of time series into consideration when robustifying the estimators. Zieliński (1999) investigated the median-unbiased estimation of the stationary AR(1) process. Molinares, et al. (2009) investigated robust estimation in long-memory processes when the data contains additive outliers.

Besides, several articles focused on the estimation of ACF of stationary time series including the work of Berkoun, et al. (2003) mentioned above. Smadi, et al. (2009) and Smadi (2013) generalized the work of Berkoun, et al. (2003) to periodic AR models. Alternatively, Hassani (2010) found that the distributions of a set of sample autocorrelations are neither independent nor identically distributed. This finding implies that the result of diagnostic check and model building based on $r_{k}$, especially in the presence of some suspect data can be quite misleading. Kan and Wang (2010) provide an algorithm for evaluating the exact distribution of the sample autocorrelations.

\section{Some properties of the ACF of AR(1) model}

Let $\left\{X_{t}\right\}$ be a stationary time series, then the auto-covariance function (ACVF) and the autocorrelation function (ACF) depend on time lag only. Based on a realization $\left\{X_{1}, \ldots, X_{n}\right\}$, the moment estimator of $\rho_{k}$ is given by $r_{k}$ defined in (5) above (Wei, 2006). For large $n, r_{k}$ is approximately normally distributed with mean $\rho_{k}$. Also, for a stationary Gaussian process, based on Bartlett (1946), Brockwell \& Davis (2002) have shown that for $k>0$ and $k+j>0$,

$$
\operatorname{Cov}\left(r_{k}, r_{k+j}\right) \cong \frac{1}{n} \sum_{i=1}^{\infty}\left\{\left(\rho_{i+k}+\rho_{i-k}-2 \rho_{i} \rho_{k}\right) \times\left(\rho_{j+k}+\rho_{j-k}-2 \rho_{j} \rho_{k}\right)\right\}
$$




\section{SMADI ET AL}

which in turn for $j=0$ reduces to

$$
\operatorname{Var}\left(r_{k}\right) \cong \frac{1}{n} \sum_{i=1}^{\infty}\left(\rho_{i+k}+\rho_{i-k}-2 \rho_{i} \rho_{k}\right)^{2}
$$

For more details on properties of $r_{k}$ for stationary time series, see Wei (2006).

Now, if $\left\{X_{t}\right\}$ follows the zero-mean AR(1) Model, as (1) with $c=0$ and $|\phi|<1$, then $\rho_{k}=\phi^{k}, k=0,1,2, \ldots$ and (8) reduces to

$$
\begin{aligned}
\operatorname{Var}\left(r_{k}\right) & \approx \frac{1}{n} \sum_{i=1}^{k} \phi^{2 k}\left(\phi^{-1}-\phi^{i}\right)^{2}+\sum_{i=k+1}^{\infty} \phi^{2 i}\left(\phi^{-k}-\phi^{k}\right)^{2} \\
& \approx \frac{1}{n}\left\{\left(1-\phi^{2 k}\right)\left(1+\phi^{2}\right)\left(1-\phi^{2}\right)^{-1}-2 k \phi^{2 k}\right\} .
\end{aligned}
$$

A further approximation of (10) gives for $k=1$ (Cryer \& Chan, 2008)

$$
\operatorname{Var}\left(r_{k}\right) \approx \frac{1-\phi^{2}}{n}
$$

So that the closer $\phi$ is to \pm 1 the more accurate the estimate of $\rho_{1}(=\phi)$ becomes. For large values of $k$, the terms in (10) involving $\phi^{k}$ could be ignored so that

$$
\operatorname{Var}\left(r_{k}\right) \approx \frac{1}{n}\left[\frac{1+\phi^{2}}{1-\phi^{2}}\right] .
$$

In Figure 1, $n \operatorname{Var}\left(r_{k}\right)$ is sketched based on (10) for some selected values of $\phi$. Notice in this figure that for $k=1$, as $|\phi|$ gets larger, $\operatorname{Var}\left(r_{k}\right)$ is decreasing. Therefore, with stronger autocorrelation among the data, the moment estimator $r_{1}$ is more accurate. The opposite happens for $k \geq 1$, that is as $|\phi|$ approaches one, $\operatorname{Var}\left(r_{k}\right)$ becomes larger. 


\section{ROBUSTNESS OF ESTIMATORS OF THE ACF OF AR(1) PROCESS}

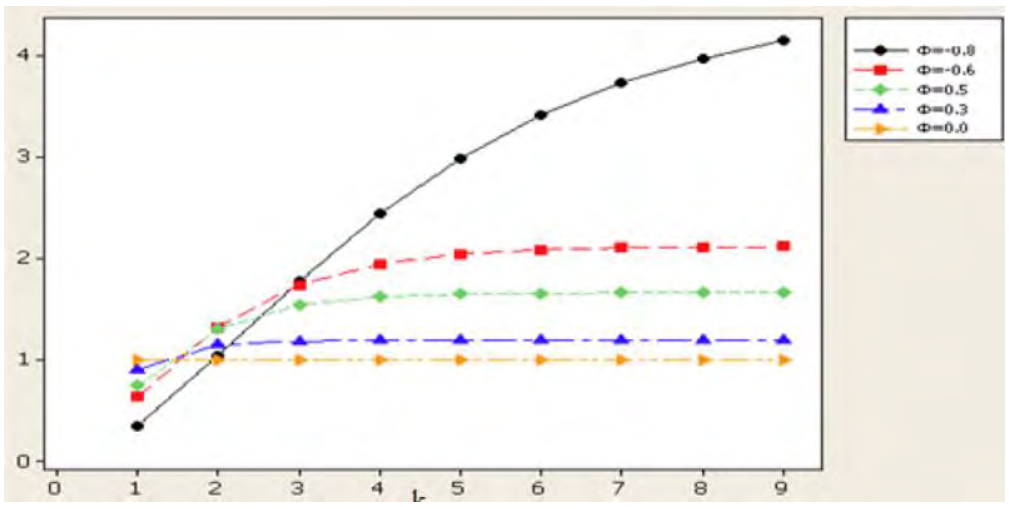

Figure 1. $n \operatorname{Var}\left(r_{k}\right)$ for the $\mathrm{AR}(1)$ model for several values of $\phi$ and $k$.

For the AR(1) model, (8) can also be simplified for general $0<k<k+j$ as (Cryer \& Chan, 2008)

$$
\operatorname{Cov}\left(r_{k}, r_{k+j}\right) \cong \frac{1}{n} \frac{\left(\phi^{j}-\phi^{2 k+j}\right)\left(1+\phi^{2}\right)}{\left(1-\phi^{2}\right)}+j \phi^{j}-(2 k+j) \phi^{2 k+j}
$$

In particular,

$$
\operatorname{Corr}\left(r_{1}, r_{2}\right) \cong 2 \phi \sqrt{\frac{1-\phi^{2}}{1+2 \phi^{2}+3 \phi^{4}}}
$$

Figure 2 shows $\left|\operatorname{Corr}\left(r_{1}, r_{2}\right)\right|$ for some selected values of $\phi$. This figure shows a stronger association between $r_{1}$ and $r_{2}$ when $|\phi|$ is closer to one. More precisely, for the $\mathrm{AR}(1)$ time series data, when $\phi \approx 1(\phi \approx-1)$ a large positive (negative) $r_{1}$ is expected to be followed by a relatively large positive (positive) $r_{2}$. This agrees with the theoretical ACF of $\mathrm{AR}(1)$ model, $\rho_{k}=\phi^{k}$, which is alternating for negative values of $\phi$.

Notice from the discussion above, that for stationary time series data, $r_{k}$ is asymptotically unbiased. The formulas for the variance and covariances among various sample autocorrelations depend mainly on the theoretical ACF of the model and they are again asymptotic. In the following example, using MonteCarlo simulation, the bias and MSE of $r_{k}$ for the AR(1) model are studied and the accuracy of the asymptotic variance of $r_{k}$ given by (11) and (12) is investigated. 


\section{SMADI ET AL}

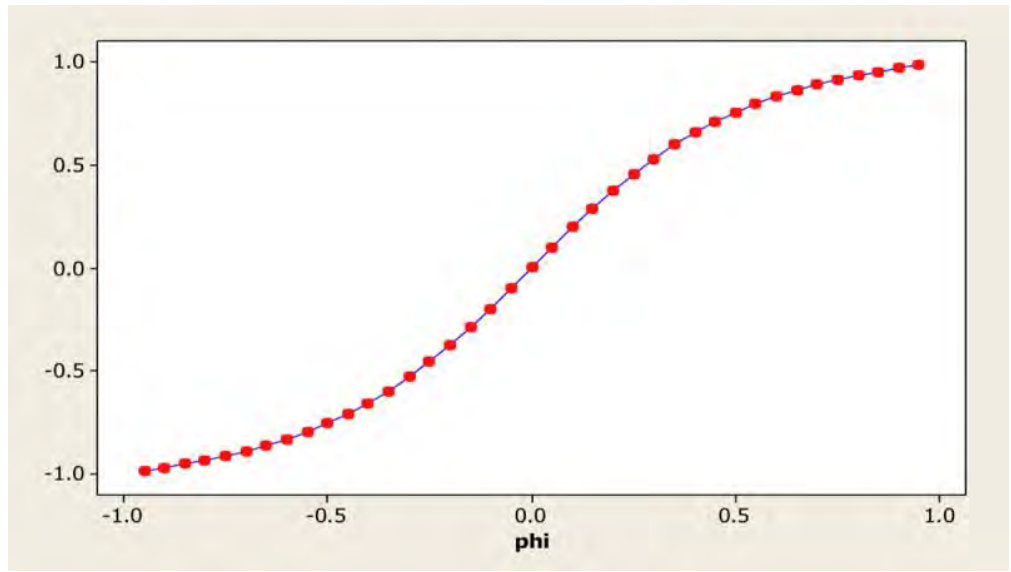

Figure 2. Asymptotic Corr $\left(r_{1}, r_{2}\right)$ for $A R(1)$ model for several values of $\phi$.

\section{Example 1}

The accuracy of the formulas for $\operatorname{Var}\left(r_{k}\right)$ given in (11) and (12) are now studied. Assuming the zero-mean AR(1) model with $\phi=-0.8,-0.5,-0.1,0.1,0.5,0.8$, one thousand realizations each of length $n=30,100$ are generated from this model assuming that the $\mathrm{WN}$ process is iid $N(0,1)$, then the sample ACF for lags $k=1, \ldots, 5$ is computed, then the sample MSE are computed (in terms of $\rho_{1}$ and its estimates $\left.r_{1(1)}, \ldots, r_{1(1000)}\right)$ as

$$
M S E=\frac{1}{1000} \sum_{i=1}^{1000}\left(\rho_{1}-r_{1(i)}\right)^{2} .
$$

finally Rel-MSE $=$ MSE $/ \operatorname{Var}\left(r_{k}\right)$ are computed, where (11) and (12) are used for $\operatorname{Var}\left(r_{k}\right)$, which in turn are sketched in Figure 3. The simulations are carried out using the R-package through the R-command sim.ARIMA

In view of Figure 3, it can be seen that Rel-MSE is close to one (which means that the asymptotic formulas in (11) and (12) become more accurate) when $\phi$ is close to zero and $\mathrm{n}$ is large. In addition, it seems that (11) underestimates the actual variance for $r_{1}$. For $k \geq 2$, the asymptotic variance is defined by (12). For $r_{2}$ with $n=100$ this formula again underestimates the actual variance but, unexpectedly, not true for $n=30$. For larger time lags, it is seen that Rel-MSE < 1 , so that (12) overestimates the actual variance of $r_{k}$. Therefore, in practice, (11) 


\section{ROBUSTNESS OF ESTIMATORS OF THE ACF OF AR(1) PROCESS}

and (12) should be used with caution as they may produce poor results depending on the type and strength of autocorrelation among data and the realization length.
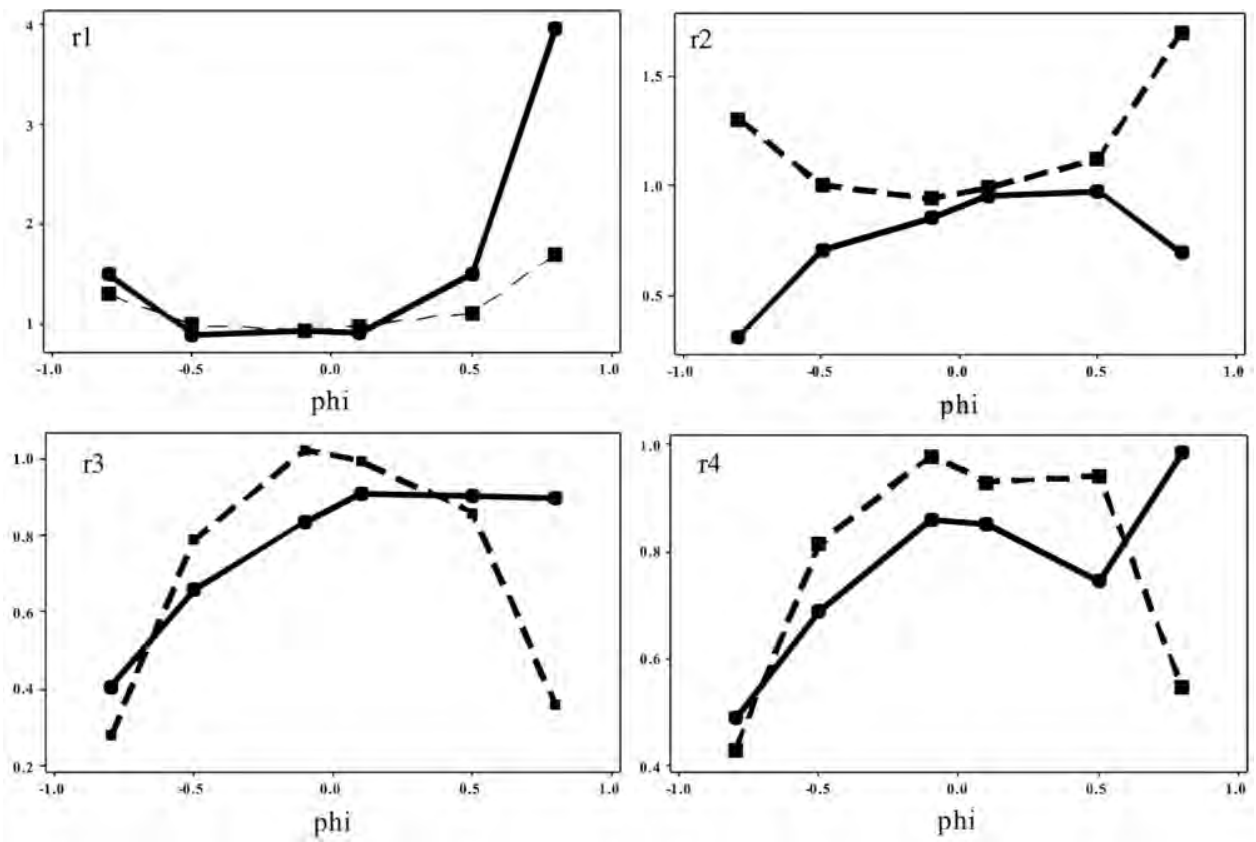

Figure 3. The Rel-MSE of $r_{k}$ for the AR(1) model for various values of $\phi(n=30: \sim$, $n=100:---)$

\section{The empirical distributions of some robust estimators of $\rho_{1}$ for the AR(1) model}

An estimation procedure is said to be robust if it is little influenced by blatant departures from assumptions. Such procedures aim to minimize the influence of outliers or departure from model assumptions while performing at the same time as well as the optimum methods when assumptions hold (Sprent and Smeeton, 2001).

In (3) and (4), two robust estimators of $\rho_{1}$ are defined due to Berkoun, et al. (2003) which have been generalized to higher time lags in (6) and (7). Recall that $\rho_{1}$ is of particular importance specially in the AR(1) model for which $\rho_{1}=\phi$. This value determines the strength and pattern of all remaining autocorrelations. Also, in many routine statistical analyses, as for instance in testing for autocorrelated 


\section{SMADI ET AL}

errors in regression analysis, only $\rho_{1}$ is usually investigated. The following example investigates the distributions of various estimators of $\rho_{1}$ for the $\operatorname{AR}(1)$ model.

\section{Example 2}

Assuming the zero-mean $\mathrm{AR}(1)$ model with $\mathrm{WN}$ following $N\left(0, \sigma^{2}\right)$, then using Monte-Carlo simulation the distributions of $r_{1}, \rho_{1}^{*}$ and $\tilde{p}_{1}$ for $\phi=0.1,0.8, n=100$ and $\sigma^{2}=1$ are compared. An r-code is written by the authors to accomplish this job. The empirical distributions of various estimators are obtained based on one thousand repetitions using the r-command (density). Results are presented graphically in Figure 4. This figure shows that the empirical distributions of various estimators are unimodal and nearly symmetric. The distribution of $\rho_{1}^{*}$ seems closer to normality than other distributions. As $\phi$ is increased from 0.1 to 0.8 , the location of various distributions is shifted up towards 0.8 . Also, the variability in the distributions of $r_{1}$ and $\rho_{1}^{*}$ is decreased more than that of the second robust estimator, $\tilde{p}_{1}$.
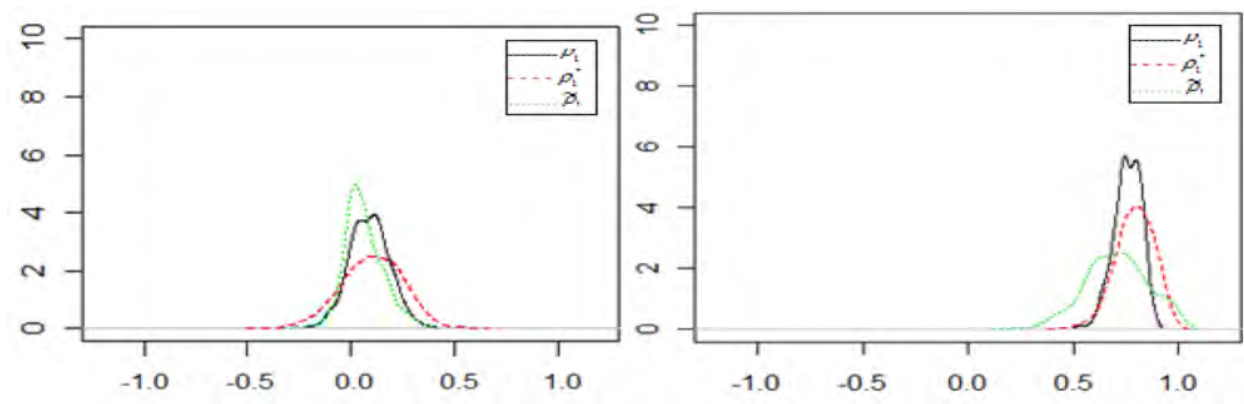

Figure 4. The empirical pdf of three estimators of $\rho_{1}$ for $\operatorname{AR}(1)$ model; for $\phi=0.1$ (left) and $\phi=0.8$ (right), $n=100$ and $\sigma^{2}=1$

In traditional time series analysis, it is usually assumed that the $\mathrm{WN}$ terms in the $\mathrm{AR}(1)$ model are iid $N\left(0, \sigma^{2}\right)$, as above. Therefore, it is crucial to explore the robustness of various estimators of the ACF if the WN terms are not normal. In the following example the empirical distributions of various estimators for $\rho_{1}$ in the AR(1) model are investigated assuming that the $\mathrm{WN}$ terms follow the normal, student-t, Cauchy and exponential. The choice of the student-t and Cauchy 


\section{ROBUSTNESS OF ESTIMATORS OF THE ACF OF AR(1) PROCESS}

distributions was to study the effect of tail-heaviness of WN distribution whereas the exponential distribution is used to study the effect of skewness of WN distribution.

\section{Example 3}
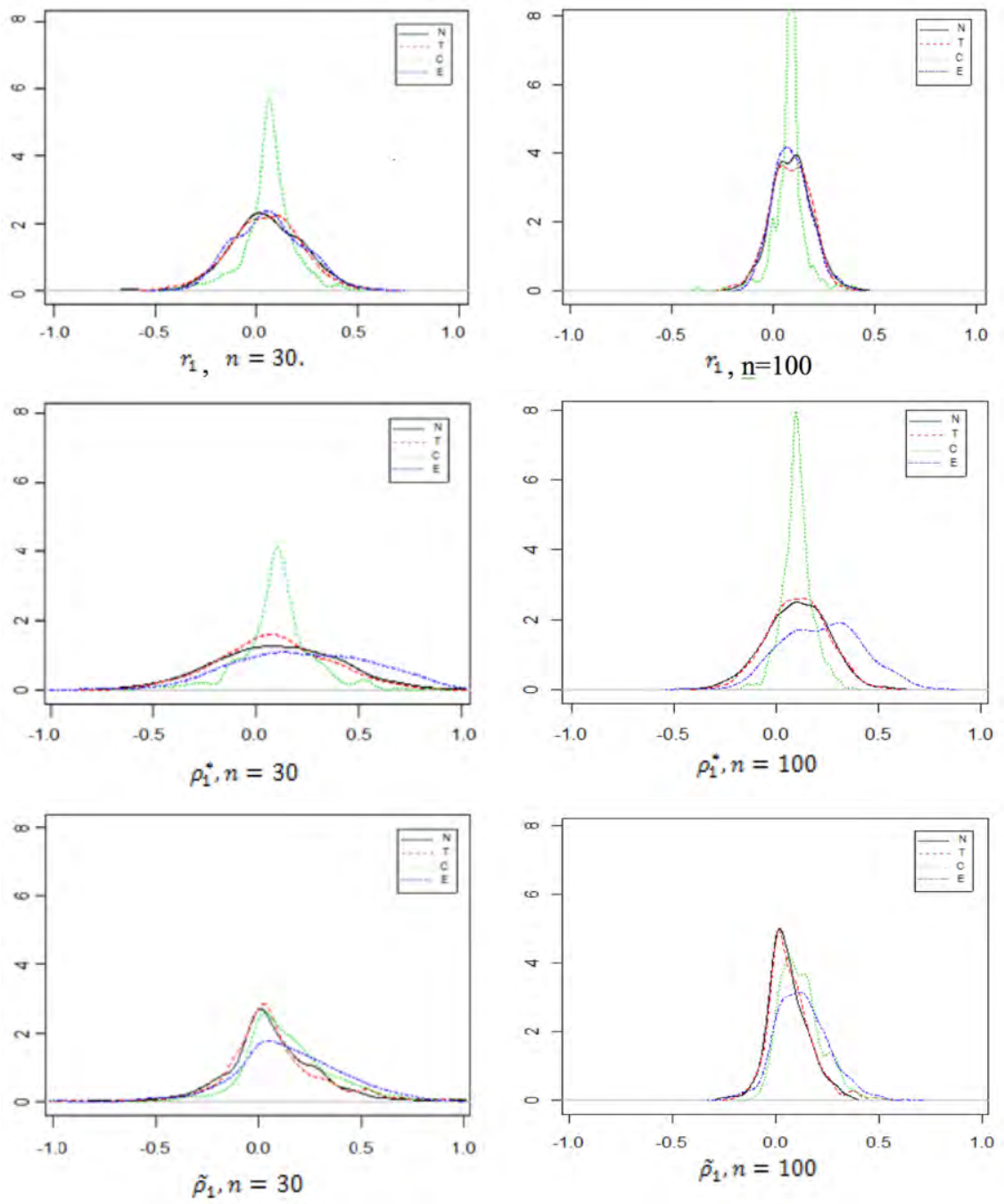

Figure 5. Empirical distributions of $r_{1}, \rho_{1}^{*}$ and $\tilde{p}_{1}$ of $\mathrm{AR}(1)$ model; for $\phi=0.1$, for various error distributions.

Assuming the zero-mean AR(1) model with $\mathrm{WN}$ following $N(0,1), \mathrm{t}_{5}$, Cauchy $(0,1)$, and (the zero-mean) $\operatorname{Exp}^{*}(1)$ (that is, the ordinary exponential distribution 


\section{SMADI ET AL}

with mean 1 but shifted left by one unit). Again, using Monte-Carlo simulation the distribution of $r_{1}, \rho_{1}^{*}$ and $\tilde{p}_{1}$ for $\phi=0.1,0.8, n=30,100$ are compared. The empirical distributions of various estimators is obtained based on 1,000 repetitions using the r-command (density). The results are summarized in Figure 5. Also, the p-values of two tests of normality for the empirical distributions, namely the Shapiro-Wilk test (SWT) and the Anderson-Darling test (ADT), are presented in Table 1. To perform these tests, the R-commands shapiro.test(X) and ad.test(x) were used which belong, respectively to the stats and nortest R-packages. A detailed account of these tests and other tests of normality is found in Thode (2002).

Table 1. Normality tests of the distributions of $r_{1}, \rho_{1}^{*}$ and $\tilde{\rho}_{1}$ of $\operatorname{AR}(1)$ model; for $\phi=0.1,0.8, n=30,100$, along various error distributions.

\begin{tabular}{|c|c|c|c|c|c|c|c|}
\hline \multirow[b]{2}{*}{$\phi$} & \multirow[b]{2}{*}{$n$} & \multicolumn{3}{|c|}{$\mathbf{N}$} & \multicolumn{3}{|c|}{$\mathbf{T}$} \\
\hline & & $r_{1}$ & $\rho_{1}^{*}$ & $\tilde{\rho}_{1}$ & $r_{1}$ & $\rho_{1}^{*}$ & $\tilde{\rho}_{1}$ \\
\hline \multirow{2}{*}{\multicolumn{2}{|c|}{30}} & 0.0700 & 0.0458 & $2.50 \mathrm{E}-11$ & 0.5264 & 0.9339 & $5.61 \mathrm{E}-12$ \\
\hline & & 0.0076 & 0.0259 & $2.20 \mathrm{E}-16$ & 0.5338 & 0.9008 & $2.20 \mathrm{E}-16$ \\
\hline \multirow{2}{*}{\multicolumn{2}{|c|}{100}} & 0.4270 & 0.7529 & $7.76 \mathrm{E}-11$ & 0.3117 & 0.7291 & $2.20 \mathrm{E}-16$ \\
\hline & & 0.2414 & 0.7529 & $1.39 \mathrm{E}-15$ & 0.6098 & 0.9912 & $2.20 \mathrm{E}-16$ \\
\hline \multirow{2}{*}{\multicolumn{2}{|c|}{30}} & $2.20 \mathrm{E}-16$ & $2.20 \mathrm{E}-16$ & $2.20 \mathrm{E}-16$ & $2.20 \mathrm{E}-16$ & $2.20 \mathrm{E}-16$ & $2.20 \mathrm{E}-16$ \\
\hline & & $2.20 \mathrm{E}-16$ & $2.20 \mathrm{E}-16$ & $2.20 \mathrm{E}-16$ & $2.20 \mathrm{E}-16$ & $2.20 \mathrm{E}-16$ & $2.20 \mathrm{E}-16$ \\
\hline \multirow{2}{*}{\multicolumn{2}{|c|}{100}} & $9.23 E-12$ & 5.63E-09 & 1.96E-07 & $1.28 \mathrm{E}-11$ & 1.19E-08 & 7.80E-07 \\
\hline & & $6.05 \mathrm{E}-14$ & $5.12 \mathrm{E}-07$ & $2.00 \mathrm{E}-03$ & $1.06 \mathrm{E}-13$ & $3.48 \mathrm{E}-11$ & $2.44 \mathrm{E}-03$ \\
\hline \multirow[b]{2}{*}{$\phi$} & \multirow[b]{2}{*}{$n$} & \multicolumn{3}{|c|}{ C } & \multicolumn{3}{|c|}{ E } \\
\hline & & $r_{1}$ & $\rho_{1}^{*}$ & $\tilde{\rho}_{1}$ & $r_{1}$ & $\rho_{1}^{*}$ & $\tilde{\rho}_{1}$ \\
\hline \multirow{2}{*}{\multicolumn{2}{|c|}{30}} & $9.54 \mathrm{E}-16$ & $2.20 \mathrm{E}-16$ & $2.20 \mathrm{E}-16$ & 0.01542 & $1.21 \mathrm{E}-05$ & $3.35 \mathrm{E}-07$ \\
\hline & & $2.20 \mathrm{E}-16$ & $2.20 \mathrm{E}-16$ & $2.20 \mathrm{E}-16$ & 0.01491 & 3.02E-05 & $1.60 \mathrm{E}-11$ \\
\hline \multirow{2}{*}{\multicolumn{2}{|c|}{100}} & $2.20 \mathrm{E}-16$ & $3.41 \mathrm{E}-15$ & $2.20 \mathrm{E}-16$ & $6.92 \mathrm{E}-05$ & $4.12 \mathrm{E}-05$ & $9.54 \mathrm{E}-15$ \\
\hline & & $2.20 \mathrm{E}-16$ & $2.20 \mathrm{E}-16$ & $2.20 \mathrm{E}-16$ & $6.42 \mathrm{E}-05$ & 7.41E-05 & $1.43 \mathrm{E}-15$ \\
\hline \multirow{2}{*}{\multicolumn{2}{|c|}{30}} & $2.20 \mathrm{E}-16$ & $2.20 \mathrm{E}-16$ & $2.20 \mathrm{E}-16$ & $2.20 \mathrm{E}-16$ & $2.20 \mathrm{E}-16$ & $2.20 \mathrm{E}-16$ \\
\hline & & $2.20 \mathrm{E}-16$ & $2.20 \mathrm{E}-16$ & $2.20 \mathrm{E}-16$ & $2.20 \mathrm{E}-16$ & $2.20 \mathrm{E}-16$ & $2.20 \mathrm{E}-16$ \\
\hline \multirow{2}{*}{\multicolumn{2}{|c|}{$\begin{array}{l}0.8 \\
100\end{array}$}} & $2.20 \mathrm{E}-16$ & $2.20 \mathrm{E}-16$ & $2.20 \mathrm{E}-16$ & $3.29 \mathrm{E}-10$ & $1.74 \mathrm{E}-13$ & 3.77E-09 \\
\hline & & $2.20 \mathrm{E}-16$ & $2.20 \mathrm{E}-16$ & $6.57 \mathrm{E}-15$ & 2.16E-08 & $3.61 \mathrm{E}-14$ & $3.47 \mathrm{E}-06$ \\
\hline
\end{tabular}

In view of Table 1 , it can be seen that the majority of distributions are far from normality, especially when the $\mathrm{WN}$ distribution is far from normality. The 
departure from normality is specifically seen for smaller $\mathrm{n}$ as well as larger $\phi$. The normality assumption is validated only with $\phi=0.1$, for $r_{1}$ and $\rho_{1}^{*}$ along normal and $\mathrm{t}$ WN distributions. In summary, results indicated that the assumption of normality for any of the estimators of $\rho_{1}$ considered here is mostly invalid. This result agrees with those of Hassani (2010).

As far as Figure 5 is considered, it can be seen that the empirical distribution of $r_{1}$ is not affected by the WN distribution, except the Cauchy case which showed a much higher kurtosis than other distributions. This is true for $n=30$ and 100. A nearly similar conclusion is seen for $\rho_{1}^{*}$. For $\tilde{p}_{1}$, no significant differences are seen, especially for $n=30$, among its empirical distributions in terms of WN distribution including the Cauchy distribution, whereas all distributions here show some positive skewness. In overall, it seems that $\tilde{p}_{1}$ is less affected by the change of WN distribution as compared to other estimators.

\section{Bias and MSE for various estimators of ACF of AR(1) with Gaussian errors}

Now, go back to the case of normal WN distribution and again the AR(1) model. The objective is to study the precision and accuracy of various estimators for $\rho_{k}$ defined in (5) - (7). Example 1 defined the empirical MSE of $r_{1}$. Similarly, the bias of $r_{1}$ is defined as

$$
\text { Bias }=\frac{1}{1000} \sum_{i=1}^{1000}\left(\rho_{1}-r_{1(i)}\right) \text {. }
$$

Because interest is in determining the bias and MSE for various time lags and $\phi$ which in turn change the theoretical autocorrelations, the better comparable measures are computed, namely the relative bias (RB) and relative root MSE (RRMSE) defined as

$$
R B(\hat{\theta})=\frac{B(\hat{\theta})}{\theta}
$$

and 


\section{SMADI ET AL}

$$
\operatorname{RRMSE}(\hat{\theta})=\frac{\sqrt{M S E(\hat{\theta})}}{|\theta|} .
$$

Table 2. The RB and RRMSE for various estimators of the ACF of $A R(1)$ model.

\begin{tabular}{|c|c|c|c|c|c|c|}
\hline \multirow[b]{2}{*}{$\phi$} & \multirow[b]{2}{*}{ Est } & \multicolumn{5}{|c|}{$n=30$} \\
\hline & & 1 & 2 & 3 & 4 & 5 \\
\hline \multirow{6}{*}{-0.8} & \multirow{2}{*}{$r_{k}$} & 0.079 & 0.172 & 0.232 & 0.313 & 0.355 \\
\hline & & $(0.168)$ & $(0.340)$ & $(0.485)$ & $(0.665)$ & $(0.827)$ \\
\hline & \multirow{2}{*}{$p_{k}^{*}$} & 0.038 & 0.056 & 0.078 & 0.111 & 0.153 \\
\hline & & $(0.235)$ & $(0.418)$ & $(0.626)$ & (0.899) & (1.197) \\
\hline & \multirow{2}{*}{$\tilde{p}_{k}$} & 0.127 & 0.176 & 0.199 & 0.218 & 0.244 \\
\hline & & $(0.331)$ & $(0.484)$ & $(0.651)$ & $(0.871)$ & (1.136) \\
\hline \multirow{6}{*}{-0.5} & \multirow{2}{*}{$r_{k}$} & 0.062 & 0.240 & 0.234 & 0.565 & $(0.013)$ \\
\hline & & $(0.301)$ & $(0.792)$ & (1.528) & (3.131) & (5.987) \\
\hline & \multirow{2}{*}{$p_{k}^{*}$} & 0.028 & 0.081 & 0.188 & 0.203 & 0.013 \\
\hline & & $(0.494)$ & (1.219) & $(2.520)$ & (5.219) & $(11.136)$ \\
\hline & \multirow{2}{*}{$\tilde{p}_{k}$} & 0.201 & 0.282 & 0.355 & 0.394 & 0.262 \\
\hline & & $(0.561)$ & (1.061) & (2.043) & (4.089) & (8.723) \\
\hline \multirow{6}{*}{-0.1} & \multirow[b]{2}{*}{$r_{k}$} & $(0.268)$ & 2.610 & $(20.900)$ & 287.000 & $(1960.000)$ \\
\hline & & $(1.755)$ & (17.020) & $(168.500)$ & (1711.700) & $(16763.100)$ \\
\hline & \multirow{2}{*}{$p_{k}^{*}$} & $(0.040)$ & $(0.950)$ & 9.000 & 137.000 & 1080.000 \\
\hline & & $(2.888)$ & $(27.530)$ & $(291.900)$ & $(3108.100)$ & $(31686.000)$ \\
\hline & \multirow{2}{*}{$\tilde{p}_{k}$} & 0.213 & $(0.130)$ & 6.800 & 130.000 & 300.000 \\
\hline & & $(2.211)$ & $(22.270)$ & $(226.100)$ & (2370.700) & $(25059.900)$ \\
\hline \multirow{6}{*}{0.1} & \multirow[b]{2}{*}{$r_{k}$} & 0.442 & 4.180 & 47.100 & 295.000 & 3280.000 \\
\hline & & $(1.732)$ & (17.970) & $(175.500)$ & $(1702.900)$ & $(17406.900)$ \\
\hline & \multirow{2}{*}{$p_{k}^{*}$} & 0.023 & 0.790 & 17.000 & (147.000) & $(470.000)$ \\
\hline & & $(2.827)$ & $(29.780)$ & $(302.700)$ & $(3028.200)$ & $(32542.300)$ \\
\hline & \multirow{2}{*}{$\tilde{p}_{k}$} & 0.247 & 0.450 & 11.400 & (138.000) & $(920.000)$ \\
\hline & & (2.112) & (22.270) & $(230.000)$ & (2267.200) & $(24819.300)$ \\
\hline \multirow{6}{*}{0.5} & \multirow[b]{2}{*}{$r_{k}$} & 0.209 & 0.526 & 0.966 & 1.634 & 2.906 \\
\hline & & $(0.388)$ & $(0.932)$ & (1.787) & (3.256) & (6.336) \\
\hline & \multirow{2}{*}{$p_{k}^{*}$} & 0.060 & 0.076 & 0.198 & 0.195 & 0.221 \\
\hline & & $(0.507)$ & (1.257) & $(2.747)$ & (5.445) & (11.250) \\
\hline & \multirow{2}{*}{$\tilde{p}_{k}$} & 0.218 & 0.270 & 0.289 & 0.350 & 0.346 \\
\hline & & $(0.562)$ & (1.077) & (2.172) & (4.443) & (9.230) \\
\hline \multirow{6}{*}{0.8} & \multirow[b]{2}{*}{$r_{k}$} & 0.200 & 0.395 & 0.582 & 0.780 & 0.986 \\
\hline & & $(0.272)$ & $(0.508)$ & $(0.721)$ & (0.945) & (1.170) \\
\hline & \multirow{2}{*}{$p_{k}^{*}$} & 0.035 & 0.073 & 0.094 & 0.105 & 0.127 \\
\hline & & $(0.225)$ & $(0.428)$ & (0.657) & (0.891) & (1.242) \\
\hline & \multirow{2}{*}{$\tilde{p}_{k}$} & 0.129 & 0.182 & 0.197 & 0.214 & 0.224 \\
\hline & & $(0.329)$ & $(0.497)$ & $(0.673)$ & $(0.863)$ & (1.134) \\
\hline
\end{tabular}




\section{ROBUSTNESS OF ESTIMATORS OF THE ACF OF AR(1) PROCESS}

Table 2, cont.

\begin{tabular}{|c|c|c|c|c|c|c|}
\hline \multirow[b]{2}{*}{$\phi$} & \multirow[b]{2}{*}{ Est } & \multicolumn{5}{|c|}{$n=100$} \\
\hline & & 1 & 2 & 3 & 4 & 5 \\
\hline \multirow{6}{*}{-0.8} & \multirow{2}{*}{$r_{k}$} & 0.026 & 0.056 & 0.077 & 0.104 & 0.119 \\
\hline & & $(0.086)$ & $(0.176)$ & $(0.273)$ & $(0.387)$ & $(0.514)$ \\
\hline & \multirow{2}{*}{$p_{k}^{*}$} & 0.012 & 0.024 & 0.027 & 0.024 & 0.011 \\
\hline & & $(0.127)$ & $(0.243)$ & $(0.363)$ & $(0.504)$ & $(0.680)$ \\
\hline & \multirow{2}{*}{$\tilde{p}_{k}$} & 0.110 & 0.183 & 0.237 & 0.245 & 0.251 \\
\hline & & $(0.229)$ & $(0.357)$ & $(0.465)$ & $(0.555)$ & $(0.669)$ \\
\hline \multirow{6}{*}{-0.5} & \multirow{2}{*}{$r_{k}$} & 0.013 & 0.064 & 0.068 & 0.190 & 0.061 \\
\hline & & $(0.173)$ & $(0.458)$ & $(0.933)$ & $(1.986)$ & (3.945) \\
\hline & \multirow{2}{*}{$p_{k}^{*}$} & 0.006 & $(0.001)$ & 0.000 & 0.061 & $(0.125)$ \\
\hline & & $(0.268)$ & $(0.655)$ & $(1.353)$ & $(2.889)$ & (5.778) \\
\hline & \multirow{2}{*}{$\tilde{p}_{k}$} & 0.237 & 0.352 & 0.414 & 0.411 & 0.346 \\
\hline & & $(0.381)$ & $(0.632)$ & $(0.990)$ & $(1.893)$ & (3.592) \\
\hline \multirow{6}{*}{-0.1} & \multirow{2}{*}{$r_{k}$} & $(0.037)$ & 0.970 & $(10.800)$ & 124.000 & $(720.000)$ \\
\hline & & $(0.964)$ & $(10.200)$ & $(100.000)$ & $(1000.000)$ & $(10148.900)$ \\
\hline & \multirow{2}{*}{$p_{k}^{*}$} & 0.029 & 0.680 & (8.800) & (17.000) & 520.000 \\
\hline & & (1.565) & $(15.750)$ & $(154.600)$ & $(1486.600)$ & $(16522.700)$ \\
\hline & \multirow{2}{*}{$\tilde{p}_{k}$} & 0.414 & 0.640 & (4.700) & $(20.000)$ & $(10.000)$ \\
\hline & & $(1.058)$ & $(9.327)$ & $(93.270)$ & $(866.000)$ & $(9434.000)$ \\
\hline \multirow{6}{*}{0.1} & \multirow{2}{*}{$r_{k}$} & 0.158 & 1.360 & 14.000 & 102.000 & 1460.000 \\
\hline & & $(0.990)$ & $(10.050)$ & $(97.470)$ & $(964.400)$ & $(10148.900)$ \\
\hline & \multirow{2}{*}{$p_{k}^{*}$} & 0.046 & 0.100 & (2.700) & 21.000 & 470.000 \\
\hline & & (1.533) & $(16.210)$ & $(156.800)$ & $(1612.500)$ & $(16673.300)$ \\
\hline & \multirow{2}{*}{$\tilde{p}_{k}$} & 0.438 & 0.330 & $(3.700)$ & $(6.000)$ & 80.000 \\
\hline & & (1.058) & $(9.434)$ & $(96.950)$ & $(927.400)$ & $(9848.900)$ \\
\hline \multirow{6}{*}{0.5} & \multirow{2}{*}{$r_{k}$} & 0.053 & 0.141 & 0.278 & 0.549 & 0.986 \\
\hline & & $(0.183)$ & $(0.478)$ & $(1.002)$ & (1.992) & $(3.880)$ \\
\hline & \multirow{2}{*}{$p_{k}^{*}$} & 0.008 & 0.009 & $(0.010)$ & 0.122 & 0.064 \\
\hline & & $(0.271)$ & $(0.650)$ & $(1.415)$ & $(2.920)$ & $(5.831)$ \\
\hline & \multirow{2}{*}{$\tilde{p}_{k}$} & 0.242 & 0.352 & 0.397 & 0.406 & 0.480 \\
\hline & & $(0.381)$ & $(0.620)$ & $(1.024)$ & $(1.979)$ & (3.677) \\
\hline \multirow{6}{*}{0.8} & \multirow{2}{*}{$r_{k}$} & 0.052 & 0.108 & 0.168 & 0.230 & 0.302 \\
\hline & & $(0.098)$ & $(0.200)$ & $(0.309)$ & $(0.431)$ & $(0.574)$ \\
\hline & \multirow{2}{*}{$p_{k}^{*}$} & 0.009 & 0.010 & 0.029 & 0.018 & 0.014 \\
\hline & & $(0.123)$ & $(0.228)$ & $(0.348)$ & $(0.473)$ & $(0.660)$ \\
\hline & \multirow{2}{*}{$\tilde{p}_{k}$} & 0.115 & 0.190 & 0.235 & 0.256 & 0.275 \\
\hline & & $(0.229)$ & $(0.347)$ & $(0.451)$ & $(0.530)$ & (0.639) \\
\hline
\end{tabular}

Therefore, assuming the same model and settings in Example 1, and based on one thousand realizations, the RB and RRMSE for $r_{k}, \rho_{k}^{*}$ and $\tilde{\rho}_{k}$ are computed and then summarized in Table 2. The main advantage of adopting $R B$ and RRMSE is that they can be used to compare any two cases (cells) within Table 2, regarding the value of $\phi, n$ or estimator. 


\section{SMADI ET AL}

The first conclusion from Table 2 is that, for fixed $\phi$ and $n$ the RB and RRMSE increase for all estimators as the time lag $k$ is increasing. The RB and RRMSE also increase for all estimators as $|\phi|$ approaches zero. Thus, it may be concluded that with stronger autocorrelation among data, all estimators perform better than for weaker autocorrelation. It can also be seen that the RB and RRMSE for negative values of $\phi$ are slightly smaller than their corresponding positive values.

As the sample size increases, it can be seen that the RB and RRMSE are decreasing for $r_{k}$ and $\rho_{k}^{*}$. For $\rho_{k}^{*}$, the RRMSE is decreasing along $n$, but the RB shows no clear pattern.

When $|\phi|$ is large, no big differences are seen in RB and RRMSE for various estimators, while discrepancies appear as $|\phi|$ gets closer to zero. In overall, it seems that $\rho_{k}^{*}$ is better than other estimators in terms of RB and $r_{k}$ is better than other estimators in terms of RRMSE.

\section{Conclusions}

This study considered the statistical properties of the ACF of the AR(1) model, beginning with instigating some asymptotic formulas for the variances and covariance for the sample ACF $\left(r_{k}\right)$ for AR(1) model. It was noticed that some asymptotic formulas for $\operatorname{Var}\left(r_{k}\right)$ are not accurate, especially for strong autocorrelation $(|\phi|$ closer to one).

Later, the empirical distributions for three estimators of the first lag

autocorrelation, $r_{1}, \rho_{1}^{*}$ and $\tilde{\rho}_{1}$, were studied, where the later two estimators are two robust estimators of $\rho_{1}$. These distributions are investigated for various error distributions. It is noticed that the empirical distributions of $r_{1}$ and $\rho_{1}^{*}$ are only affected when the error distribution is Cauchy, while the third estimator $\tilde{\rho}_{1}$ is found more robust in this regard. Conversely, it is seen that the majority of empirical distributions are far from normality for all estimators.

Earlier the accuracy and precision of higher lags estimators of $\rho_{k}$, were studied, namely $r_{k}, \rho_{k}^{*}$ and $\tilde{\rho}_{k}$ for the $\mathrm{AR}(1)$ model with normal errors. It is seen that, all estimators were more accurate and precision for $|\varphi|$ closer to one and small time lags. Besides, the RB and RRMSE dramatically increase when $\phi$ is closer to zero and large time lags. In overall, $r_{k}$ perform better than other 


\section{ROBUSTNESS OF ESTIMATORS OF THE ACF OF AR(1) PROCESS}

estimators in terms of RRMSE while $\rho_{k}^{*}$ is better than other estimators in terms of RB.

Finally, this study indicates that the moment estimator of the ACF of AR(1) model is an important tool in the identification and estimation of such models. It seems that it behaved well when the error of distributions is non-normal. However, the accuracy and precision of the moment ACF may suffer for weaker autocorrelations among data and higher time lags. It seems that more effort is needed following the current work, either regarding the model type of data or improving the accuracy and precision of the sample ACF of data.

\section{References}

Akaike, H. (1974). Markovian representation of stochastic processes and its application to the analysis of autoregressive moving average processes. Annals of the Institute of Statistical Mathematics, 26: 363-387.

Bartlett, M. S. (1946). On the theoretical specification of sampling properties of auto-correlated time series. Journal of the Royal Statistical Society, Series B, 8: 27-41.

Beguin, J. M., Gourieroux, C., \& Monfort, A. (1980). Identification of a mixed autoregressive moving average process: The corner method. In O. D. Anderson (Ed.). Time series (pp. 423-436). Amsterdam: North-Holland.

Berkoun, Y., Fellag, H., \& Zieliński, R. (2003). Robust Testing serial correlation in AR (1) processes in the presence of a single additive outlier. Communications in Statistics, Theory and Methods, 32(8): 1527-1540.

Box, G., Jenkins, G., \& Reinsel, G. (1994). Time series analysis, forecasting and control, $3^{\text {rd }}$ Edition. New York, NY: Prentice-Hall.

Brockwell, P., \& Davis, R. (2002). Introduction of time series and forecasting, $2^{\text {nd }}$ Edition. New York, NY: Springer-Verlag.

Bustos, O. H., \& Yohai, V. J. (1986). Robust estimates of ARMA models. Journal of the American Statistical Association, 81: 69-155.

Cleveland, W. S. (1972). The inverse autocorrelations of a time series and their applications. Technometrics, 14: 277-293.

Cryer, J., \& Chan, K. (2008). Time series analysis with applications in $R$, $2^{\text {nd }}$ Edition. New York, NY: Springer. 


\section{SMADI ET AL}

Denby, L., \& Martin, R. D. (1979). Robust estimation of the first-order autoregressive parameter. Journal of the American Statistical Association, 74: 140-146.

Gray, H. L., Kelley, G. D., \& McIntire, D. D. (1978). A new approach to ARMA modeling, Communications in Statistics, 87: 1-77.

Haddad, J. N. (2000). On robust estimation in the first-order autoregressive processes, Communications in Statistics, Theory and Methods, 29(11): 45-54.

Hassani, H. (2010). A note on the sum of the sample autocorrelation function. Journal Physica A: 1601-1606.

Huber, P. J. (1964). Robust estimation of a location parameter. The Annals of Mathematical Statistics, 35: 73-101.

Hurwicz, L. (1950). Least-squares bias in time series. In T. C. Koopmans (Ed.). Statistical inference in dynamic economic models, (pp. 365-383). New York, NY: Wiley and Sons.

Kan, R., \& Wang, X. (2010). On the distribution of the sample autocorrelation coefficients. Econometrics, 154: 101-121.

Molinares, F., Reisen, V., \& Neto, F. (2009). Robust estimation in longmemory processes under additive outliers. Journal of Statistical Planning and Inference, 139: 2511-2525.

Smadi, A. A. (2013). A Comparison of Some Estimators of the Seasonal ACF for Various PAR Models. Accepted in the Journal of Applied Probability and Statistics, January, 2013.

Smadi, A., Abu-Affouna, N., \& Al-Quraan, A. (2009). Robust estimation of the seasonal autocorrelation of the PAR (1) model. Jordan Journal of Mathematics and Statistics, 2(2): 105-118.

Sprent, P., \& Smeeton, N. C. (2001). Applied nonparametric statistical methods, $3^{\text {rd }}$ Edition. Boca Raton, FL: Chapman and Hall/CRC.

Thode Jr., H. C. (2002). Testing for normality. New York, NY: Marcel Dekker.

Wei, W. W. (2006). Time series analysis, univariate and multivariate methods, $2^{\text {nd }}$ Edition. Boston, MA: Addison-Wesley.

Zieliński, R. (1999). A median-unbiased estimator of the AR(1) coefficient. Journal of Time Series Analysis, 20(4): 477-481. 